Katarzyna Sikorska-Bujnowicz*

\title{
FREMDSPRACHENPROBLEME ALS MITTEL DES HUMORISTISCHEN AM BEISPIEL DER DEUTSCHEN TÜRKENWITZE
}

\section{Zielsetzung}

In diesem Beitrag wird versucht, die Frage nach der Rolle der fehlerhaften sprachlichen Formen als Mittel des Humoristischen zu beantworten. Am Beispiel der deutschen Türkenwitze wird gezeigt, welche die den Ausländern die meisten Schwierigkeiten bereitenden Strukturen der deutschen Sprache als Motive und Zielscheiben im Türkenwitz zu nennen sind.

Der Analyse werden die nach dem Kriterium der eingesetzten fehlerhaften Formen als Mittel des Humoristischen ausgewählten Sketchtexte aus der Comedyshow „Was guckst du?!“ und die den Internetseiten entstammenden längeren Witze $^{1}$ unterzogen.

In diesem Beitrag werden nur die 6 repräsentativsten Beispiele angeführt und besprochen. Im Zentrum des Interesses stehen die von den Autoren eingesetzten, auf die Sprachschwierigkeiten zurückgehenden fehlerhaften sprachlichen Formen. Alle zitierten Witze stehen demzufolge in der nicht korrigierten Originalform. Es konnte gezeigt werden, welche Subgruppen der morphologischen Fehler und die der syntaktischen relativ häufig auftreten. Da die für die Zwecke dieses Beitrags durchgeführte Analyse keine Gesprächsanalyse ist und die verschrifteten bzw. geschriebenen Witze anbetrifft, wurde auf die phonetische Ebene als Quelle der als Mittel des Humoristischen zu verstehenden Fehler verzichtet.

\footnotetext{
* Dr. Katarzyna Sikorska-Bujnowicz, Universität Łódź, Institut für Germanische Philologie, Pomorska 171/173, 90-236 Łódź. E-Mail: k.sikorska_bujnowicz@poczta.onet.pl

${ }^{1}$ Als Quellen dienten folgende Internetseiten: http:/www.grocceni.com/witze/witz0859.html, http://www.witze1000.de/italienerwitze, http://www.lachmeister.de/lustige-witze/italiener/index. html, http://www.witze.woxikon.de/witze-uni-und-schule/320, http://www.witzdestages.net/witze/laender-witze/, http://www.witze.net/araber-witze, http://www.witze-ueber-witze.de/nationalitaetenwitze.html
} 


\section{Zum Phänomen von „Was guckst du?!“}

„Was guckst du?" scheint wahrhaftig ein Beitrag zur Verständigung in Deutschland zu sein, der mit umwerfendem Humor den Menschen und Nationen Charakteristika lässt und sie zugleich zu einer (TV) Völkerfamilie eint frei nach dem Motto: Gemeinsam lacht sich`s besser. ${ }^{1}$

Die im deutschen Fernsehen in den 90er Jahren des 20. Jahrhunderts laufende Comedyshow „Was guckst du?!“ gilt bis heute noch als erste deutsche Ethnocomedy, was auf die dem Rezipienten angebotene Art Humor zurückgeht. Die mit viel Distanz zu sich selbst als Vertreter der Ausländergesellschaft in Deutschland präsentierte Problematik bedeutete für den Moderator Kaya Yanar seinen Aufstieg in der Fernsehwelt. Das Publikum hat den von ihm mit viel Biss, aber zugleich mit viel Sympathie jeder in der Show dargestellten und auszulachenden Nation gegenüber angebotenen Humor anerkannt ${ }^{2}$ und auf diese Weise konnten auch die alltäglichen Probleme der sich in Deutschland zurückzufinden versuchenden Ausländer zwar im Zerrspiegel, aber doch präsentiert werden, sodass zum ersten Mal so offensichtlich, obwohl auf eine außergewöhnliche Art und Weise die Ausländerprobleme so offen angesprochen wurden.

Für die von den Autoren der Sendung ausgedachten Sketchtexte sind vor allem im Rahmen der sprachlichen Mittel des Humoristischen die aus den Sprachschwierigkeiten resultierenden Missverständnisse charakteristisch. Die damit zusammenhängenden, einerseits von den Gestalten der Witzszenen unabsichtlich begangenen, andererseits aber von den Autoren mit Absicht und zum Zwecke des Witzigen eingesetzten Fehler werden somit zum wichtigsten sprachlichen Element der von „Was guckst du?" angebotenen Texte. Somit stehen die fehlenden Sprachkenntnisse im Vordergrund und die den Einwanderern viele Schwierigkeiten bereitende deutsche Sprache wird zur Quelle des Witzigen.

In der von Yanar moderierten Sendung wird nicht selten zur Gegenüberstellung Deutsch vs. Türkisch bzw. Arabisch gegriffen. Die Autoren bedienen sich dabei der als broken German zu bezeichnenden Sprache, unter der eine Mischung aus Deutsch, Türkisch oder Arabisch zu verstehen ist. Die mit fehlerhaften Formen gekennzeichnete Variante des Deutschen wird auf diese Weise zum Erkennungszeichen der Sendung.

Die von „Was guckst du?“ angebotene Humorart kann auch in den im Internet zu findenden Witzen wiedergefunden werden, sodass eine Analyse der humoristischen Texte unter dem Aspekt der fehlerhaften Formen als Mittel des Humoristischen möglich war.

${ }^{1}$ Vgl. W. Deutschmichel: ,, Was guckst Du? “, von: http://www.mi.niedersachsen.de/portal/live. php?navigation_id=14791\&article_id=60594\&_psmand=33, Zugriff 2015.

${ }^{2}$ Unter den für seine Show verliehenen Preise sind folgende zu nennen: Deutscher Fernsehpreis in der Kategorie Beste Comedy (2001), Deutscher Comedypreis in der Kategorie Beste Comedysendung (2001), Goldene Romy in der Kategorie Beste Programmidee (2001) und CIVIS Medienpreis (2001), Deutscher Comedypreis für das beste Live-Programm 2013 und Bester Komiker 2014. 


\section{Einige Bemerkungen zum Ethnowitz als Sprachwitz}

Die Analyse des Humoristischen geht jedes Mal auf die von Raskin und Attardo im Jahre 1991 formulierte Allgemeine Theorie des Verbalen Humors (General Theory of Verbal Humour) zurück. Die von den Autoren aufgestellte These von den sich in jedem witzigen Text versteckenden, sich mithilfe des Nonverbalen erweitern lassenden Inhalten kann im Rahmen der Analyse der Sketchtexte bestätigt werden. Mit im Spiel stehen dann zwei Kontexte - der sprachliche und der außersprachliche. Die von den Rezipienten erwartete Kenntnis des jeweiligen Kulturkreises und der für die konkrete nationale Gruppe charakteristischen Mentalität bewirkt das erwünschte Interpretieren der angebotenen Witze.

Die jeden Witz als Text definierenden Bestandteile, d.h. ihr Thema, das Motiv, die Zielscheibe und die Pointe hängen in erster Linie mit der Annahme zusammen, dass alles Präsentierte schon beim Lesen korrekt dekodiert werden kann.

Für die Gruppe der Ethnowitze ${ }^{3}$ ist auch die für ihr richtiges Verstehen von dem Rezipienten verlangte sprachliche Wissen, d.h. die Sprachkompetenz charakteristisch. ${ }^{4}$ Bei Driessen (1999) wird eine besondere Rolle der Sprachkenntnisse beim richtigen Verstehen der humoristischen Texte betont. Mit dem Wissen aus diesem Bereich hängt die Möglichkeit zusammen, die bisher unbekannte Kulturwelt näher kennen zu lernen und, was nicht außer Acht gelassen werden darf, alle im Witz verborgenen Inhalte zu finden und zu dekodieren.

Die von den Witzautoren mit Absicht eingesetzten fehlerhaften Formen werden nicht als störende Elemente betrachtet, weil sie als Motiv und als Zielscheibe gelten können.

Die auf diese Weise entstehende scherzhafte Kommunikation ist voll von morphologischen Fehlern oder den im Bereich der Syntax. Wegen ihres Bezugs auf die Witzform sind die auf den genannten Ebenen auftretenden Probleme bei der Analyse der geschriebenen Witze ausschlaggebend.

Kaya Yanar betont in einem der zahlreichen Interviews $(2008)^{5}$, wie wichtig für die richtige Kommunikation die Sprachkenntnisse sind, auch im Rahmen des Scherzhaften:

Das Wort „Merde“ heißt auf Deutsch „Scheiße“, aber wie hört sich das an:

Scheiße! (...) Aber Merde könnte auch, wenn man kein Französisch kann, eine Vorspeise sein.

${ }^{3}$ Dem deutschen Ethnowitz habe ich u.a. meine 2016 erschienene Monographie gewidmet: vgl. K. Sikorska-Bujnowicz (2016), Zum deutschen Ethnowitz. Eine korpusgestützte Analyse, Łódź.

${ }^{4}$ Bei Raskin (1985) wird der äußerst wichtige Einfluss der Sprache der jeweiligen sozialen Gruppe auf den humoristischen Text betont.

5 Vgl. mehr dazu: J. Wittner (2008), Interview Kaya Yanar. Merde in Germany (=http://www. stern.de/kultur/tv/interview-kaya-yanar-merde-in-germany-3090962.html, Zugriff: 2015). 
Das weist darauf hin, dass fehlende Kenntnisse der fremden Lexik einerseits zu den Missverständnissen führen, andererseits aber auch als Mittel des Humoristischen gebraucht werden können.

Die durchgeführte Analyse der ausgewählten Witze zeigt, dass fehlende Sprachkenntnisse und die mit ihnen verbundenen und aus ihnen resultierenden Missverständnisse an erster Stelle unter den als sprachliche Mittel eingesetzten Elementen stehen.

\section{Ergebnisse der Analyse}

Die Analyse der ausgewählten Sketchtexte aus „Was guckst du?“ und der den deutschen Internetseiten entstammenden Türkenwitze unter dem Aspekt der dort festzustellenden fehlerhaften sprachlichen Formen umfasst die aus einer Unmenge Witze über Türken stammenden, repräsentativsten humoristischen Texte, in denen eben wegen ihrer längeren Form zahlreiche Fehler verschiedener Art zu finden sind.

\subsection{Türkenwitze aus „Was guckst du?!“}

(1) Umfrage auf der Straße

D: Entschuldigung, wir machen eine Unterschriftenaktion zum Thema „Ausländer". Was sagen Sie zu den Ausländern?

T: Bitte, (1.1.) müsse nach Hause gehen!

D: Genau, müssen nach Hause gehen. Bitte unterschreiben Sie! Danke schön! Günther....

1.1. falsche Modalverbform *müsse, verstanden als müssen

(2) Ein Ausländer befragt die Passanten

Kaya: (2.1.) Entschuldigen, ich habe eine Frage..

K: (2.2.a) Wo... kann man... hier gut...

P1: Langsam...

$\mathrm{K}:$... (2.2.b) Popel essen?

$\mathrm{K}$ : (2.3.) Wo ist hier... die Toilette... machen?

P2: (2.4.) Machen nichts. Machen nichts. 
K: Doch, doch!

P2: $\quad$ Sie müssen zur Toilette.

$\mathrm{K}: \quad$ Hose

P2: (2.5.) Wenn Sie nicht rechtzeitig auf die Toilette, dann geht es in die Hose.

K: (2.6.) Entschuldigen. (2.7.) Wo... kann man hier in den Rhein schiffen?

P1: Da kommt unten aus, aus eine Säule

P1: Ja, Hohe Straße, ja..

K: Säule??, essen, (2.8.) nichts Säule

P2: Da ist die Toilette.

K: (2.9.) Dort Hose

P2: Da ist die Toilette.

$\mathrm{K}$ : OK, danke schön!!

2.1. fehlendes Pronomen in der Höflichkeitsform *Entschuldigen

2.2. falsch gebrauchtes Wort Popel

2.3. *Toilette machen anstelle von auf die Toilette gehen

2.4. falsches Negationswort nichts anstelle von nicht

2.5. fehlendes Prädikat im Nebensatz

2.6. *Entschuldigen, fehlendes Pronomen in der Höflichkeitsform

2.7. Gebrauch eines veralteten Verbs schiffen

2.8. falsches Negationswort nichts anstelle von nicht

2.9. Wortgruppe ohne Sinn

(3) Radio Süleyman 2

S: Hallo, Leute! Hier ist wieder Sprechradio Süleyman. Natürlich (3.1.) mit meine beste Freund Süleyhund. Scheiße! Muss mal wieder Gassi gehen. Braucht Auslauf. Also mach schnell. Erster Anrufer

A1: Hallo. Süleymann! Ich bin traurig.

S: $\quad$ Traurig? Es ist ein Scheißname! (3.2.) Aber was geht, Traurig?

A1: Nein, du verstehst mich nicht! Ich heiße Thomas und bin traurig. Ich lerne einfach keine Frau kennen, weil ich immer Angst habe, dass sie nur mein Geld wollen, ich bin nämlich sehr reich.

S: $\quad$ Keine Angst, wenn du erst eine Frau hast, bist du bald nicht mehr reich...!

A1: Ich will keine Frau, die nur auf Materielles aus ist. Ich habe sogar schon einmal überlegt, mein Geld einfach wegzuschmeißen.

S: $\quad$ Was??? Mach keine Dummheiten, helfe ich dir, bist du alles los - Probleme und Geld. Scheiße!!!

A3: Guten Tag, hier spricht die Wachtmeisterin Hümmer. Uns liegt eine Anzeige wegen Umweltverschmutzung vor.

S: $\quad$ Umweltverschmutzung? Ich bin doch kein Umweltverschmutzer. (3.3.) 
Umweltverschmutzer ist Mann, der Sache wegwirft oder... Moment!

S: $\quad$ Traurig? Ich hab eine gute Idee für dich. Wirfst du doch Geld weg, packst du alles in großen Sack, wirfst du (3.4.) rückwärts von Rheinbrücke.

A1: Was??? Von mir aus...Und dann lerne ich eine Frau kennen?

S: $\quad J a$, (3.5.) vertraust du mir! Wachtmeisterin. (3.6.) Habe ich gehört, steht Mann am Rheinbrücke und wirft Müllsack in Fluss. Das ist ein Umweltverschmutzer, darfst du ihn festnehmen, mich nicht!

S: Thomas, (3.7.) verspreche ich dich, wenn du den Sack geworfen hast, kommt nette Frau auf dich zu und nimmt dich mit, auch ohne Geld!

A1: Ohne? Danke, Süleyman!(...)

3.1. falsche Deklinationsformen des Possessivpronomens und des Adjektivs *mit meine beste Freund Süleyhund

3.2. * Aber was geht, Traurig? anstelle von Aber was geht ab?

3.3. fehlerhafte Formen:

3.3.1. fehlender Artikel *ist Mann

3.3.2. fehlender Artikel *Sache wegwirft

3.4. fehlender Artikel *von Rheinbrücke

3.5. falsch gebildete Imperativform *vertraust du mir!

3.6. fehlerhafter Satz:

3.6.1. Fehler in der Wortfolge *Habe ich gehört

3.6.2. fehlender Artikel *steht Mann

3.6.3. falscher Artikel *am Rheinbrücke

3.6.4. fehlender Artikel *wirft Müllsack

3.6.5. fehlender Artikel *in Fluss

3.7. fehlerhafte Formen:

3.7.1. falsche Form des Personalpronomens *verspreche ich dich

3.7.2. fehlender Artikel *kommt nette Frau

\subsection{Türkenwitze aus dem Internet}

(4) Ein Türke wird von den E-Werken angestellt, um die Zähler abzulesen. Er kommt an die erste Adresse auf seiner Liste, eine Frau öffnet die Tür. Der Türke fragt sie: (4.1.) „Wie viel Nummern du haben gemacht bei Licht?““ Die Frau empört: „Egon, komm mal schnell, hier steht ein Türke und beleidigt mich!““

Egon kommt, breit wie ein Schrank: „Was willst du?“ Der Türke: (4.2.) „Wie viel Nummern du haben gemacht bei Licht?“ Egon brüllt laut: (4.3.) „Willst du ,n paar auf die Fresse?“ Der Türke: (4.4.) „Wenn du mir nicht sagen, wie viel Nummern du haben gemacht bei Licht, ich dir schneiden (4.5.) Strippe ab, und deine Frau muss nehmen Kerze..." 
4.1.1. Fehler in der Wortfolge *Wie viel Nummern du haben gemacht bei Licht?, *ich dir schneiden Strippe ab

4.1.2. falsche Konjugationsform *du haben gemacht

4.1.3. Spiel mit der Grundbedeutung des Wortes Nummer als Zahl und seiner saloppen Bedeutungsvariante, d.h. Nummer als Geschlechtsverkehr 4.2. Wiederholung der Frage mit fehlerhaften Formen

4.3. Fragesatz mit der umgangssprachlichen Lexik ein paar auf die Fresse (bekommen)

4.4. Konditionalsatz mit fehlerhaften Formen:

4.4.1. falsche Konjugationsform *du nicht sagen, *du haben gemacht, *ich dir schneiden...ab

4.4.2. Fehler in der Wortfolge *Wenn du mir nicht sagen, *wie viel du.. haben gemacht bei Licht, *ich dir schneiden ...ab, *deine Frau muss nehmen Kerze

4.4.3. fehlender Artikel *ich dir schneiden Strippe ab, *deine Frau muss nehmen Kerze

4.5. im Kontext versteckte Information als Übertragung der Bedeutung von Strippe als Schnur auf Penis

(5) Hier der Feuerwehr-Alarmplan auf Deutsch und Türkisch:

\section{(5.1.) DEUTSCH: VERHALTEN IM BRANDFALL:}

1. Ruhe bewahren.

2. Gefährdete Personen evakuieren

3. Feuerwehr alarmieren.

4. Wenn möglich, Brand bekämpfen.

5. Fluchtwege benützen.

6. Feuerwehr einweisen.

7. Notruf:

Feuerwehr: 115.

Polizei: 112.

Rettung: 118.

\section{(5.2.) TÜRKISCH: WAS, WENN KRASS VIEL HEIß DA:}

1. Du nix laufen in Kreis, verstehst du.

2. Du tragen Özgür aus Barracka, wo is nix gut.

3. Du konkret schreien nach Mann in rote Auto.

4. Wenn du können, du mit Wassa korrekt spritz machen.

5. Du schicken andere Mann aus Barracka. 
6. Du sagen Mann in rote Auto, wo is konkret heiß.

7. Telefon wo hilft gleich:

Mann in rote Auto: 115.

Mann in grüne Auto: 112.

Mann in weiße Auto: 118.

5.1. hochdeutsche Variante im Spiel, Infinitivgruppen

5.2. türkisch-deutsche Variante der Informationstafel, fehlerhafte Formen:

5.2.1. *krass viel heiß

5.2.2. falsche Konjugationsformen *du nix laufen, *du tragen, *is nix gut, *du schreien, *du können, *du schicken, *du spritz machen, *du schicken, *du sagen, * is konkret heiß

5.2.3. falsche Deklinationsformen des Adjektivs, $3 \mathrm{x} *$ in rote Auto, *andere Mann, *in grüne Auto, *in weiße Auto

5.2.4. fehlende Artikel *in Kreis, * aus Barracka, *nach Mann, *mit Wassa

5.2.5. Gebrauch der phonetischen Variante *Wassa

5.2.6. falsches Wort *Barracka anstelle von Barracke

5.2.7. fehlerhafter Satz *wo hilft gleich

5.2.8. falsche Lexik *mit Wassa korrekt spritz machen anstelle von mit Wasser begießen

5.2.9. falsches Negationswort nix anstelle von nicht

(6) Erkan telefoniert mit seinem Chef:

(6.1.) „Du Chef, (6.2.) ich Hand- Kopf- Fußweh haben, ich nicht arbeiten kommen können!“ Sagt der Chef: „Na gut, aber ich gebe dir einen Tipp, wenn mir mal was weh tut, gehe ich zu meiner Frau und lass mir einen blasen! Danach bin ich eigentlich immer gleich wieder fit!“ Erkan sagt „Okay“ und legt auf. Nach einer Weile ruft Erkan wieder seinen Chef an und sagt: „Du Chef, du recht haben, mir nix mehr weh tut, ... und eines noch, du schönes Haus haben!“

6.1. türkisch-deutsche Anredeform

6.2.1. Fehler in der Wortfolge * ich Hand-Kopf- Fußweh haben, *ich nicht arbeiten kommen können, *du recht haben, *mir nix mehr weh tut, *du schönes Haus haben

6.2.2. falsche Konjugationsformen *du haben, *ich nicht arbeiten kommen können, *du recht haben, *du schönes Haus haben

6.2.3. falsches Negationswort *nix mehr anstelle von nichts mehr

6.2.4. falsche Lexik *Hand-Kopf-Fußweh 
In den zitierten Witzen fällt auf, dass sich die Autoren nicht nur auf eine Gruppe von Fehlern konzentrieren, sondern eher auf die Vielfältigkeit der fehlerhaften Formen hinweisen, die sie als Mittel des Humoristischen gebrauchen. Auf diese Weise sind humoristische Texte entstanden, in denen in erster Linie die fehlenden Deutschkenntnisse ausgelacht werden, sodass die Sprache an sich ihre Benutzer im Zerrspiegel darstellt.

\section{Fazit und Prognosen}

Die Analyse der angeführten Witze hat ergeben, dass in erster Linie auf die 19 festgestellten Konjugationsfehler gezeigt werden soll, z.B. *müsse, *Entschuldigen, *Habe ich gehört, du nicht sagen, *du haben gemacht, *ich dir schneiden ...ab, *du nix laufen, *du tragen, *is nix gut, *du schreien, *du können, *du schicken, *du spritz machen, *du sagen, * is konkret heiß, *du haben, *ich nicht arbeiten kommen können, *du recht haben, *du schönes Haus haben. Diese hängen des Weiteren mit den Deklinationsfehlern zusammen, sowohl in Bezug auf den Artikelgebrauch (14 Fälle, u.a. *ist Mann, *Sache wegwirft, *von Rheinbrükke, *wirft Müllsack, *in Fluss, *kommt nette Frau, *ich dir schneiden Strippe ab, * deine Frau muss nehmen Kerze, *in Kreis, * aus Barracka, *nach Mann, *mit Wassa) als auch auf die Deklination der Adjektive (7 Fälle, u.a. *mit meine beste Freund Süleyhund, * in rote Auto, *andere Mann, *in grüne Auto, *in weiße Auto) bzw. Pronomina (4 Fälle, u.a. *mit meine beste Freund Süleyhund, *verspreche ich dich).

Nicht ohne Bedeutung sind auch die als Mittel des Humoristischen eingesetzten Fehler im Rahmen der Syntax, wo die meisten Probleme mit der deutschen Wortfolge verbunden sind (12 Fälle, u.a.* ich Hand-Kopf-Fußweh haben, *ich nicht arbeiten kommen können, *du recht haben, *mir nix mehr weh tut, *du schönes Haus haben, *wo hilft gleich).

Falscher Gebrauch bestimmter Wortarten, vor allem der der Pronomina (4 Fälle, u.a. *verspreche ich dich, *mit meine...Freund, *Entschuldigen) und der Negationswörter (4 Fälle, u.a. nichts anstelle von nicht, *nix mehr anstelle von nichts mehr, nix anstelle von nicht) konnte auch festgestellt werden.

Es darf nicht außer Acht gelassen werden, dass unter den fehlerhaften Strukturen auch Fehler im Wortgebrauch (11 Fälle) zu finden sind, was auf die fehlenden Kenntnisse der deutschen Lexik zurückgeführt werden kann (u.a. Popel, *Toilette machen anstelle von auf die Toilette gehen, schiffen, *Aber was geht, Traurig? anstelle von Aber was geht ab?, *mit Wassa korrekt spritz machen anstelle von mit Wasser begießen, *Barracka anstelle von Barracke).

Die durchgeführte Analyse betrifft die morphologische, die syntaktische und die lexikalische Ebene. Sie kann aber zugleich als Ausgangspunkt für wei- 
tere Untersuchung des Witzigen, dann aber auf der phonetischen Ebene, verstanden werden. Dank der durchzuführenden Gesprächsanalyse können weitere, als sprachliche Mittel des Witzigen aufzufassende Fehler gefunden, identifiziert und klassifiziert werden.

Es ist an dieser Stelle nicht wegzudenken, dass der Humor auf Fehler als Thema und Zielscheibe verzichten kann, sodass zu den fehlerhaften sprachlichen Formen als Mittel des Humoristischen nicht mehr gegriffen wird. Kein Witzerzähler wird sich die Gelegenheit entgehen lassen, über andere zu lachen, auch wenn das für ihn nur echte Schadenfreude bedeuten könnte und obwohl auf diese Weise auch nicht selten die Tabugrenzen und die des guten Geschmacks überschritten werden.

\section{LITERATURVERZEICHNIS}

Attardo S., Raskin V. (1991), Script theory revis(it)ed: Joke similarity and joke representation model, In: Humor - International Journal of Humor Research, Volume 4, Issue 3-4, S. 293-349.

Attardo S. (1994), Linguistic Theories of Humour, Berlin-New York.

Attardo S. (1997), The semantic foundations of cognitive theories of humor in: Humor - International Journal of Humor Research, Volume 4, Issue 10, S. 395-420.

Attardo S. (2014), Encyclopedia of Humor Studies, Thousand Oaks CA.

Driessen H. (1999), Lachen und Feldforschung: Betrachtungen aus dem Blickwinkel der Ethnologie, In: J. Bremmer, H. Roodenburg (Hrsg.), Kulturgeschichte des Humors, Darmstadt, S. $167-185$.

Kaya Yanar erzählt: „Liebeserklärung an die Nation“, (2004), von www.kino.de. (=http://www. kino.de/news/liebeserklaerung-an-die-nation/165950, Zugriff 2005).

Raskin V. (1985), Semantic Mechanism of Humour, Dordrecht.

Raskin V. (2008) (Hrsg.), The Primer of Humor Research, Berlin.

Schröder S. (2001), Interview mit Kaya Yanar „Was willst du?!“, In: Subway Magazin, April 2001.

Sikorska K. (2009a), Zur Sprache der deutschen Comedyshow, In: W. Sadziński (Hrsg.), Acta Universitatis Lodziensis. Folia Germanica 5, Łódź, S. 29-37.

Sikorska K. (2009b), Język niemieckiego programu komediowego „Was guckst du?”, In: S. Dżereń-Głowacka, A. Kwiatkowska (Hrsg.), Humor. Teorie - praktyka-zastosowania. Zrozumieć humor, Piotrków Trybunalski, S. 161-167.

Sikorska-Bujnowicz K. (2012b), Ethnowitz als Wiedererkennungszeichen der deutschen Comedy-Sendung ,, Was guckst du? “, In: W. Sadziński, M. Gołaszewski (Hrsg.), Wechselbeziehungen zwischen Sprache, Literatur und Kultur (= Acta Universitatis Lodziensis. Folia Germanica 8), Łódź, S. 17-24.

Sikorska-Bujnowicz K. (2016), Zum deutschen Ethnowitz. Eine korpusgestützte Analyse, Łódź.

„Was guckst du?", (1995-2000), eigene Aufnahmensammlung der deutschen Fernsehsendung von SAT.1.

Wittner J. (2008), Interview Kaya Yanar. Merde in Germany (=http://www.stern.de/kultur/tv/interview-kaya-yanar-merde-in-germany-3090962.html, Zugriff 2015). 


\section{INTERNETQUELLEN}

http://www.grocceni.com/witze/witz0859.html

http://www.lachmeister.de/lustige-witze/italiener/index.html http://www.bfriends.brigitte.de/foren/ pavillon/44669-kurze-witze-69.html

http://www.witze.woxikon.de/witze-uni-und-schule/320

http://www.witzdestages.net/witze/laender-witze/

http://www.witze-ueber-witze.de/nationalitaetenwitze.html

http://www.witze-fun.de/witze/witz/4709.

Katarzyna Sikorska-Bujnowicz

\section{FOREIGN LANGUAGE PROBLEMS AS A MEANS OF BUILDING A SENSE OF HUMOUR BASED ON THE EXAMPLE OF GERMAN JOKES ABOUT TURKS}

(Summary)

This article attempts to show which incorrect forms used because of foreign language problems are considered to be means of building a sense of humour in German jokes about Turks. Analyses include selected sketches from the Comedy-show "Was guckst du?!" and jokes about Turks from websites.

Keywords: humour research, German joke about the Turks, incorrect forms as means of building a sense of humour 